\title{
Representations of interobject spatial relations in long-term memory
}

\author{
Björn Rump • Timothy P. McNamara
}

Published online: 25 September 2012

(C) Psychonomic Society, Inc. 2012

\begin{abstract}
A growing body of evidence has indicated that human spatial memory is organized in terms of a small number of reference directions and that interobject spatial relations are represented in terms of these directions (e.g., McNamara, 2003). The goal of the present experiments was to investigate whether the selection of reference directions also affects the fidelity with which interobject spatial relations are represented in memory. In two experiments, participants memorized a layout of nine objects and then performed judgments of relative direction (e.g., "Imagine you are standing at the clock, facing the book. Point to the phone.") at a remote location. Imagined heading (e.g., at the clock, facing the book) and allocentric target direction (e.g., the direction from clock to phone in the allocentric frame of reference used to define imagined heading) were manipulated independently. The results of both experiments showed that the same directions that were benefited in imagined headings were also benefited in allocentric target directions. These findings indicate that interobject spatial relations are preferentially represented when they coincide with a reference direction.
\end{abstract}

Keywords Spatial memory · Spatial representation · Reference directions $\cdot$ Interobject spatial relations

The ability to represent and remember the spatial organization of objects in the environment is crucial for many human activities. Because the concept of location is inherently relative, representing the locations of objects

B. Rump • T. P. McNamara $(\square)$

Department of Psychology, Vanderbilt University,

PMB 407817, 2301 Vanderbilt Place,

Nashville, TN 37240-7817, USA

e-mail: t.mcnamara@vanderbilt.edu requires the locations to be specified within a particular frame of reference. Various ways of classifying reference frames have been proposed (e.g., Levinson, 1996), but for the purpose of studying human spatial cognition, a distinction between egocentric and allocentric reference systems has proven to be useful (e.g., Pani \& Dupree, 1994; Tversky, Lee, \& Mainwaring, 1999). In egocentric reference systems, locations are specified with respect to an observer's body or parts of an observer's body (e.g., in retina, arm, or trunk coordinates). In allocentric reference systems, spatial relations are specified independently of the observer. An allocentric reference system can be defined, for instance, by an individual object, by a layout of objects, by cardinal directions, or by a geometrical feature of the environment.

Several converging lines of evidence have indicated that enduring memories of the locations of objects in the environment are specified, at least in part, in terms of allocentric reference systems (e.g., Burgess, 2008; Meilinger \& Vosgerau, 2010; Mou \& McNamara, 2002; Mou, McNamara, Rump, \& Xiao, 2006; Sargent, Dopkins, Philbeck, \& Modarres, 2008; Waller \& Hodgson, 2006). For example, Mou and McNamara had their participants view an array of objects from a heading of $315^{\circ}$. The participants were instructed to learn the layout along the egocentric $315^{\circ}$ axis or the nonegocentric $0^{\circ}$ axis. After learning, participants made judgments of relative direction using their memories (e.g., "Imagine you are standing at the lamp, facing the shoe. Point to the clock."). Participants who were instructed to learn the layout along the nonegocentric $0^{\circ}$ axis performed better from the $0^{\circ}$ heading than from the $315^{\circ}$ heading, whereas the opposite pattern was obtained for participants who learned the layout along the egocentric $315^{\circ}$ axis. In other words, participants in the $0^{\circ}$ group performed better from a novel heading $\left(0^{\circ}\right)$ than from the heading that they had actually experienced $\left(315^{\circ}\right)$. 
To explain these and related findings, McNamara and colleagues (e.g., McNamara, 2003; Mou \& McNamara, 2002; Mou, McNamara, Valiquette, \& Rump, 2004; Shelton $\&$ McNamara, 2001) proposed a theoretical framework for understanding human spatial memory. The theory posits that learning the layout of a novel environment involves interpreting it in terms of a spatial reference system. This process is analogous to determining the "top" of a figure, in that conceptual "north" is assigned to the layout, which in turn determines its perceived orientation (e.g., Rock, 1973). The layout is interpreted in terms of an intrinsic reference system, which is an allocentric reference system defined by the layout of the to-be-represented objects. A given collection of objects will have an infinite number of possible intrinsic reference directions, but because of the particular arrangement of the objects and because of spatial and nonspatial properties of the objects, some directions will be more salient than others, and will therefore be more likely to be selected to serve as reference directions. Features of the surrounding environment, such as the walls of a room or geographical slant (e.g., McNamara, Rump, \& Werner, 2003; Shelton \& McNamara, 2001; Schmidt \& Lee, 2006; Steck, Mochnatzki, \& Mallot, 2003), can also make some intrinsic reference directions more salient than others. Typically, egocentric experience is the dominant cue for selecting reference directions (e.g., Greenauer \& Waller, 2008), but as the results of Mou and McNamara showed, intrinsic directions that do not correspond to the study view can be preferred if they are made sufficiently salient. Once a person has represented a layout in terms of a particular intrinsic reference system, he or she usually continues to interpret the layout through this reference system, even when seeing the layout from a novel position. If, however, the novel perspective is aligned with salient directions in the environment and the layout was originally represented using a reference system that was not aligned with these directions, then the layout may be reinterpreted in terms of a new reference system (e.g., Shelton \& McNamara, 2001).

A model consistent with the proposed theoretical framework is illustrated schematically in Fig. 1. Object locations are represented in the form of a network of nodes, which are interconnected by vectors. Each node represents an object; interobject direction and distance are represented through vector direction and magnitude, respectively. Interobject directions are specified with respect to one or more reference directions, which are depicted in the figure as gray arrows. Spatial relations between some but not all pairs of objects will be represented. For simplicity, the spatial relation between only two objects is depicted in Fig. 1 (Objects 3 and 5). The employed reference directions are $0^{\circ}$ and $90^{\circ}$. The angular direction from Object 3 to Object 5 is defined with respect to the reference directions of $0^{\circ}\left(\alpha_{35}\right)$ and $90^{\circ}\left(\beta_{35}\right)$. The angle $\alpha_{35}$ is the direction from 3 to 5

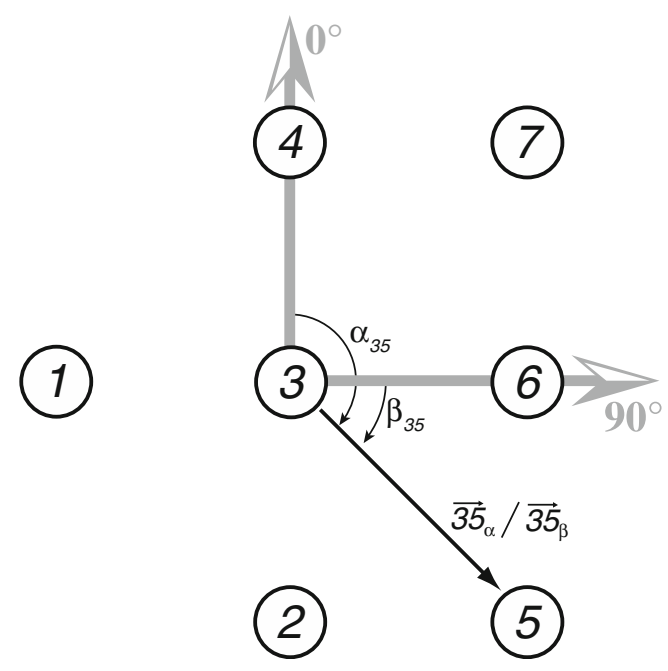

Fig. 1 Schematic depiction of an allocentric spatial representation. Numbered circles symbolize the represented objects; gray arrows symbolize reference directions. For clarity, only the represented spatial relation between Object 3 and Object 5 is shown. This spatial relation is represented with respect to the $0^{\circ}$ and $90^{\circ}$ reference directions, as indicated by the two vectors $\overrightarrow{35}_{\alpha}$ and $\overrightarrow{35}_{\beta}$. The corresponding angles of those two vectors are $\alpha_{35}$ and $\beta_{35}$, respectively

relative to the reference direction of $0^{\circ}$, not the angle formed by the Objects 4,3 , and 5 . Because this relative direction is explicitly represented in memory, it can be retrieved. Therefore, a judgment of relative direction such as "Imagine standing at 3 and facing 4. Point to 5." can be made with relative ease. In contrast, a judgment of relative direction such as "Imagine standing at 3 and facing 7. Point to 5." is difficult, because the direction from 3 to 5 is not specified in memory with respect to the direction from 3 to 7 , and therefore needs to be inferred (e.g., Klatzky, 1998).

The particular reference directions selected are important properties of a spatial representation. They determine from which headings a representation can be efficiently accessed, and from which it can not. The goal of the present experiments was to investigate whether the selection of particular reference directions has consequences that go beyond making some headings easier to access than others. More specifically, the goal was to investigate whether those interobject spatial relations that coincide with a reference direction are represented with higher fidelity or probability.

Figure 2 captures this assumption that interobject spatial relations are preferentially represented when they coincide with a reference direction. The employed reference directions in this representation are $0^{\circ}$ and $90^{\circ}$. For legibility, only the spatial relations specified relative to $0^{\circ}$ are depicted, and of those, only those that originate from Object 3. As is indicated by the solid black arrows, those interobject spatial relations that coincide with a reference direction (e.g., $\overrightarrow{34}_{\alpha}$ and $\overrightarrow{36}_{\alpha}$ ) are represented with high probability or 


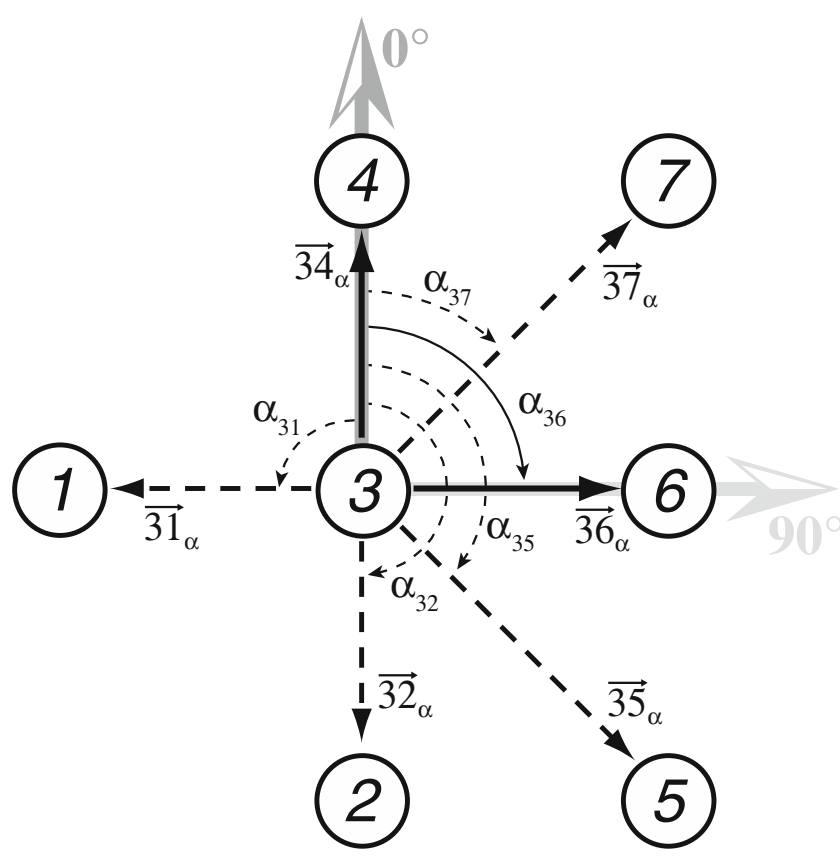

Fig. 2 A version of the model in which representations of interobject spatial relations vary in likelihood or fidelity. Spatial relations that coincide with internal reference directions (solid black arrows) are represented with higher probability or fidelity than are other spatial relations (dashed black arrows). Only the spatial relations specified relative to the $0^{\circ}$ reference direction are shown, and of those, only the ones originating from Object 3

fidelity. ${ }^{1}$ Those relations that do not coincide with a reference direction (e.g., $\overrightarrow{37}_{\alpha}$ and $\overrightarrow{35}_{\alpha}$ ) are represented with lower probability or fidelity, as indicated by the dashed black arrows. According to this model, making a spatial judgment such as "Imagine that you are standing at 3 and facing 4. Point to 6." would be relatively easy, because the spatial relations between Object 3 and Objects 4 and 6 are represented in memory, and the imagined heading is parallel to the appropriate reference direction $\left(0^{\circ}\right)$. By contrast, a judgment such as "Imagine that you are standing at 3 and facing 4. Point to 7." would be more difficult, because the spatial relation between Objects 3 and 7 is represented with lower probability or fidelity.

Preliminary evidence for the preferential representation of interobject spatial relations that coincide with a reference direction was presented by Valiquette, McNamara, and Smith (2003). In a study designed to investigate the effects of locomotion and incidental learning on the selection of reference directions, the authors discovered in post-hoc analyses that participants' pointing judgments tended to be more accurate when the direction of the target object was the

\footnotetext{
${ }^{1}$ Note that spatial relations such as $\overrightarrow{67}_{\alpha}$ (i.e., the direction from 6 to 7 , relative to $0^{\circ}$ ), if they had been included in the figure, would have also been depicted as solid black arrows, because they coincide with a reference direction, since reference directions are not fixed with respect to a particular origin.
}

same as a selected reference direction. Unfortunately, pointing direction was not varied systematically and independently of imagined heading in this study, rendering these findings inconclusive.

If interobject spatial relations that coincide with reference directions are indeed preferentially represented, for imagined headings that correspond to utilized reference directions, pointing to a target object that lies in the same direction as a reference direction should be easier than pointing to a target object that does not lie in such a direction, as described above. For judging spatial relations relative to imagined headings that do not correspond to utilized reference directions, predictions are less straightforward. For example, returning to Fig. 2, the question is whether pointing to Object 6 would still be benefited over pointing to Object 7 when the imagined heading was $135^{\circ}$ ("Imagine that you are standing at 3 and facing 5."). Our model states that in this case, spatial relations cannot be directly retrieved and need to be inferred, which is assumed to produce costs in terms of accuracy and latency. The specifics of such inferential processes are not yet specified in the model. One possible mechanism is that the target object direction is first retrieved relative to a reference direction and then transformed in some manner to match the imagined heading (e.g., via processes functionally equivalent to vector addition). To the extent that processes used to estimate the target's direction are independent of those needed to estimate the imagined heading, the benefit in retrieving spatial relations that coincide with a reference direction should cascade through the system and be observable in improved performance for allocentric target directions that coincide with reference directions, even for misaligned imagined headings. An absence of such a benefit would indicate that the target direction is not retrieved independently of other processes needed to make the pointing judgment (e.g., Roskos-Ewoldsen, McNamara, Shelton, \& Carr, 1998).

In both of the present experiments, participants studied a layout of nine objects from a single viewing position and subsequently completed a set of judgments of relative direction in a remote room. Imagined heading and allocentric target direction, defined as the direction of the target object relative to an allocentric frame of reference, were varied independently. Performance across imagined headings was used to assess which reference directions participants had apparently selected for representing the layout. The goal was then to test whether those directions also showed a benefit for allocentric target direction, and if they did, whether this effect would be present for all imagined headings, or only for those that corresponded to a reference direction.

The precise factors that govern the selection of reference directions are not fully understood at this point. As was discussed previously, egocentric experience is typically the 
dominant cue, unless the environment provides a highly salient direction that differs from the egocentrically experienced direction (Mou \& McNamara, 2002). Under certain conditions, more than one reference direction can be selected, but it appears that in this case, the selected reference directions have to be opposite or orthogonal to each other, and each needs to be perceived as very salient in the environment (e.g., Mou, Zhao, \& McNamara, 2007). In Experiment 1 , we tried to increase the saliency of those directions that were aligned with the dominant geometric structure of the environment (e.g., the walls of the surrounding room or the intrinsic structure of the object layout) by disorienting participants after entering the study room and leading them on a convoluted path to their appropriate study position. The assumption was that these procedures would require participants to completely reorient at the study position, which in turn would make them pay particular attention to the geometric structure of the surrounding environment, and especially the directions emphasized by the organization of the object layout and by the walls of the surrounding room (e.g., Cheng \& Newcombe, 2005; Holmes \& Sholl, 2005; Kelly, McNamara, Bodenheimer, Carr, \& Rieser, 2008; Mou et al., 2006; Waller \& Hodgson, 2006; Wang \& Spelke, 2000). In Experiment 2, participants were neither disoriented nor led on a convoluted path to their appropriate study position. We conjectured that these conditions would reduce the likelihood of participants representing the object layout using all four directions that were aligned with the dominant geometric structure of the environment as reference directions.

\section{Experiment 1}

In Experiment 1, participants were disoriented after entering the study room and led on a convoluted path to their appropriate study position. We anticipated that the need to completely reorient would encourage participants to pay particular attention to the room geometry, thereby making it likely that they would represent the object layout relative to the four directions that were aligned with the walls of the surrounding room and emphasized in the organization of the object layout.

\section{Method}

\section{Participants}

A group of 32 Vanderbilt University undergraduates (16 female, 16 male) participated in this experiment in return for either credit toward a course requirement or $\$ 10-\$ 15$. Three additional participants were excluded from the analyses because of excessive errors in their pointing judgments.

\section{Materials and design}

A layout of nine objects was constructed. The objects were selected on the basis of constraints that they be visually distinct, approximately comparable in size, and not share any obvious semantic associations. The objects were placed on a $3.3 \times 3.3 \mathrm{~m}$ mat, which occupied one half of the $5 \times$ $7 \mathrm{~m}$ enclosing room. Participants studied the layout of the objects from a single view $\left(0^{\circ}\right.$ or $\left.180^{\circ}\right)$, whereas the location of the mat depended on the study view that was assigned to a particular participant (see Fig. 3). At both locations, the mat was oriented to be congruent with the surrounding room, and the orientation of each object on the mat was random. Both study views were aligned with the walls of the room, the edges of the mat, and a salient intrinsic axis in the layout (e.g., book-brush-cap, shoe-can-banana).

Each test trial was constructed from the names of three objects in the layout and required participants to point to a target object as if standing in a particular position within the layout (e.g., "Imagine you are standing at the book and facing the cap. Point to the phone."). The first two objects established an imagined heading (e.g., book and cap), and the third object specified the target (e.g., phone). The primary independent variables were imagined heading and allocentric target direction. Eight equally spaced directions $\left(0^{\circ}\right.$ to $315^{\circ}$, in $45^{\circ}$ steps clockwise) were used for both variables, resulting in 64 different trial types. Two trials were presented for each trial type, for a total of 128 trials.

\section{Procedure}

The participants were randomly assigned to the two study view groups, with the constraint that each group contained equal numbers of males and females.

Before entering the study room, participants received instructions that they would have to learn the locations of a set of objects. It was emphasized that learning the layout very well was important and that this would make the subsequent spatial memory test easier. After being blindfolded, participants were led into the room and disoriented through a sequence of five large alternating left and right turns. They were then led on a convoluted path to their assigned study position, where the blindfold was removed and the objects were named in random order by the experimenter to avoid confusion in the subsequent pointing judgments. The participants were then allowed to study the object layout for $60 \mathrm{~s}$ before they had to close their eyes and point to individual objects, named in a random order by the experimenter. This sequence of studying and pointing was repeated three times, after which participants were offered additional time to study the layout (most chose an additional $10-15 \mathrm{~s}$ ). When participants indicated that they had learned the layout sufficiently well, they were 
Fig. 3 The two study view conditions used in Experiment 1. a $0^{\circ}$ study view; $\mathbf{b} 180^{\circ}$ study view. Objects were placed on a square mat within a rectangular room. Participants entered the room blindfolded through the door at the bottom left, were disoriented (circular arrows), and were led on a convoluted path (dashed lines) to their appropriate study position $\left(0^{\circ}\right.$ and $180^{\circ}$ arrows)
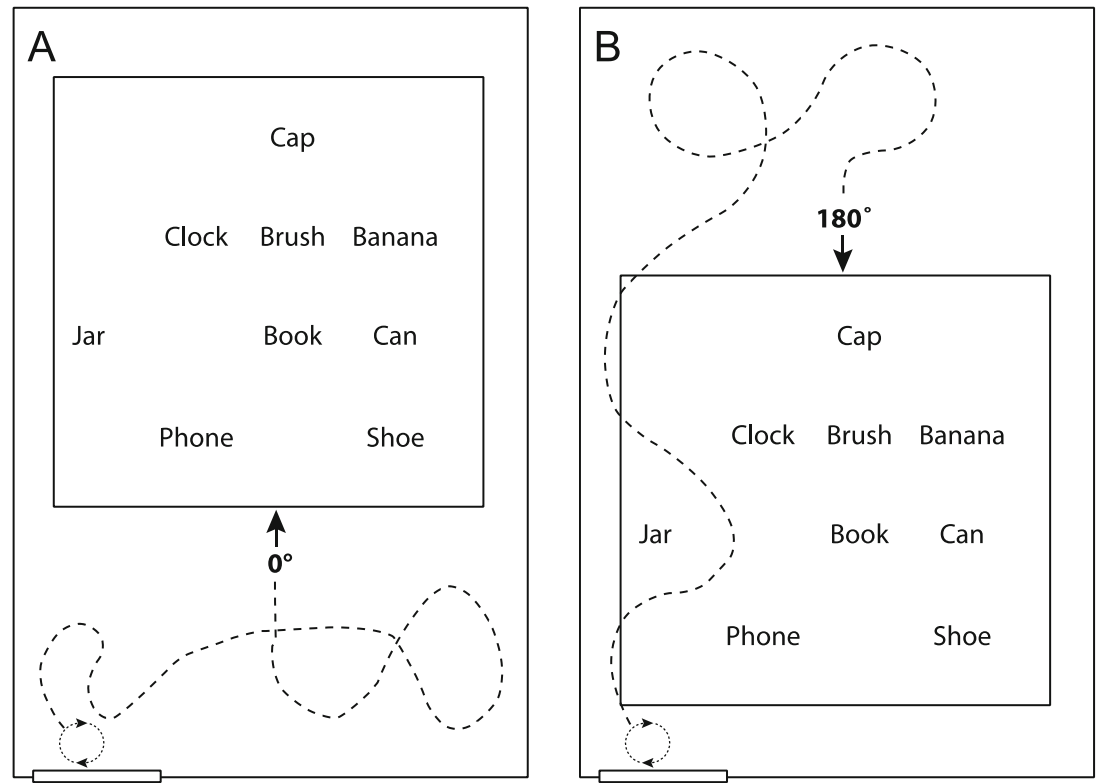

blindfolded and disoriented again and were led on a convoluted path out of the room.

Testing took place in a separate room in the same building. The participants first received instructions on the task and were then presented with four practice trials involving buildings on the Vanderbilt campus to familiarize them with the testing procedure. Participants initiated each trial by pressing a joystick button and were then presented with the trial information as text on a computer screen (e.g., "Imagine you are standing at the book and facing the cap. Point to the phone.”), which remained visible until the end of the trial. Once they had determined the direction of the target object relative to the imagined position, they pointed a Mac MouseStick II analog joystick in that direction, which terminated the trial. The joystick allowed for precise recording of both the angular error and the latency of the pointing response. Participants were instructed to emphasize accuracy over speed. They were told that they could take breaks between trials as desired. The typical duration of the experiment was 60-70 min.

\section{Results}

Three participants exhibited average absolute pointing errors greater than $50^{\circ}$ and were excluded from the following analyses. Absolute pointing errors were analyzed in a mixed-model analysis of variance (ANOVA) with terms for gender, study view $\left(0^{\circ}\right.$ or $\left.180^{\circ}\right)$, imagined heading $\left(0^{\circ}\right.$ to $315^{\circ}$ in $45^{\circ}$ steps $)$, and allocentric target direction $\left(0^{\circ}\right.$ to $315^{\circ}$ in $45^{\circ}$ steps). Imagined Heading and Allocentric Target Direction were within-participants factors. Because of the large number of effects, all tests were conducted at an alpha level of .01. Latencies showed the same general pattern as the absolute pointing errors, indicating that there were no speed-accuracy trade-offs. In the interest of brevity, only the results for pointing errors are reported.

Mean absolute pointing errors are plotted in Fig. 4 as a function of imagined heading and study view. The main effect of imagined heading was significant, $F(7,196)=21.28, M S E=$ $335.05, p<.001$, as was the Imagined Heading $\times$ Study View interaction, $F(7,196)=3.85, M S E=335.05, p<.001$. The patterns for the two study view groups, however, differed only in phase: When imagined heading was coded relative to each study view, rather than in absolute terms, the Imagined Heading $\times$ Study View interaction was not reliable, $F(7,196)<1$, $M S E=335.05$. Performance on the aligned imagined headings

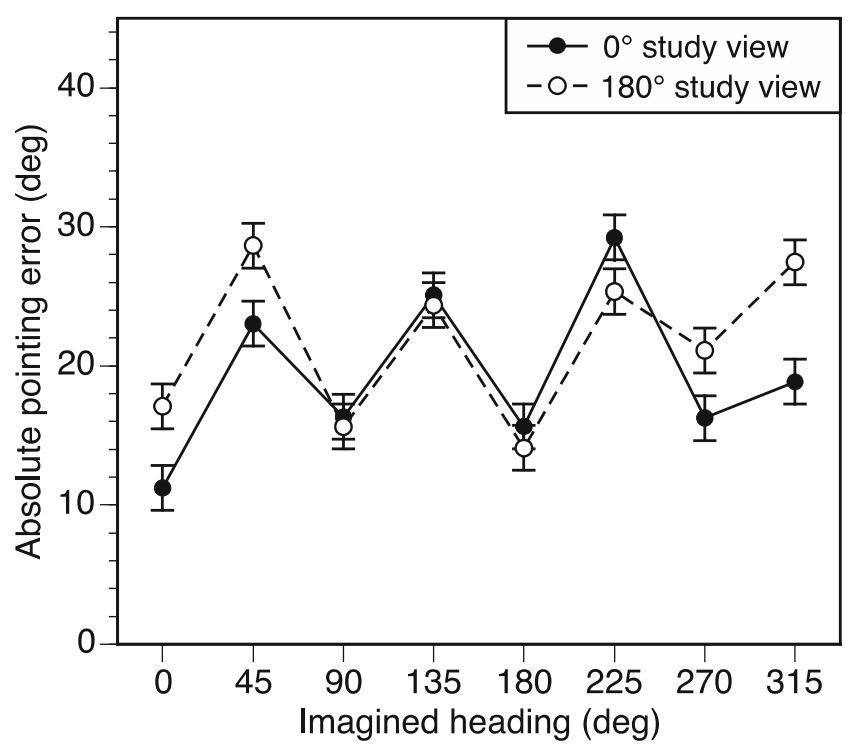

Fig. 4 Angular errors in judgments of relative direction as a function of imagined heading and study view in Experiment 1. Error bars are confidence intervals corresponding to \pm 1 standard error of the mean, as estimated from the analysis of variance 
(i.e., the imagined headings that were aligned with the dominant geometric structure of the environment: $0^{\circ}, 90^{\circ}, 180^{\circ}$, and $270^{\circ}$ ) was better than performance on the misaligned imagined headings in both the $0^{\circ}$ study view group, $F(1,196)=64.44$, $M S E=335.05, p<.001$, and the $180^{\circ}$ study view group, $F(1$, $196)=68.56, M S E=335.05, p<.001$. In each study view group, pointing errors were lower for the study view than for the other aligned headings, although these effects were of marginal statistical significance [for $0^{\circ}, F(1,196)=6.74$, $M S E=335.05, p=.011 ;$ for $180^{\circ}, F(1,196)=4.24, M S E=$ $335.05, p=.041]$.

The effect of allocentric target direction was significant, $F(7$, 196) $=7.00, M S E=310.04, p<.001$, but allocentric target direction did not interact with study view, $F(7,196)<1, M S E=$ 310.04 , nor were any higher-order interactions involving the two factors significant, $p s \geq .044$. Therefore, for the following analyses of allocentric target direction, we collapsed across study views. Allocentric target direction did, however, interact with imagined heading, $F(49,1372)=3.17, M S E=271.57, p<$ .001 . Visual inspection of the interaction indicated that a sawtooth pattern across allocentric target directions was evident for aligned but not for misaligned imagined headings. This pattern is clear in Fig. 5, which plots mean absolute angular errors in pointing as a function of allocentric target direction, separately for aligned and misaligned imagined headings. For aligned imagined headings $\left(0^{\circ}, 90^{\circ}, 180^{\circ}\right.$, and $\left.270^{\circ}\right)$, performance was better for aligned than for misaligned allocentric target directions, $F(1,1568)=104.14, M S E=276.38, p<.001$.

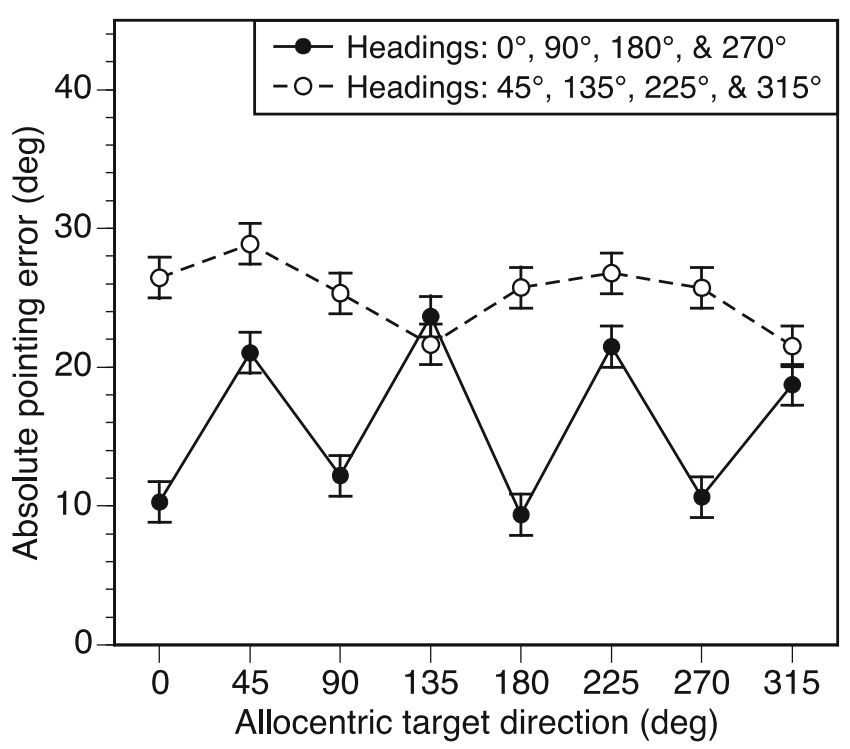

Fig. 5 Angular errors in judgments of relative direction as a function of allocentric target direction and imagined heading in Experiment 1. The solid line corresponds to imagined headings that were aligned with the dominant geometric structure of the learning environment; the dashed line corresponds to imagined headings that were not so aligned. Error bars are confidence intervals corresponding to \pm 1 standard error of the mean, as estimated from the analysis of variance
When participants imagined facing one of the misaligned headings $\left(45^{\circ}, 135^{\circ}, 225^{\circ}\right.$, and $\left.315^{\circ}\right)$, no such effect was present, $F$ $(1,1568)=1.13, M S E=276.38, p=.288$. On average, the performance for misaligned allocentric target directions within aligned headings was still better than the performance for misaligned headings overall (in Fig. 5, the peaks of the sawtooth function are on average below the dashed line), $F(1$, $1764)=19.56, M S E=282.90, p<.001$. No other statistically reliable effects were obtained.

To account for the possibility that the selection of reference directions was not as consistent across participants as the combined data suggest, we conducted an additional analysis, in which we estimated the selected reference directions at the level of the individual participant. For each participant, we started with the imagined heading on which the participant performed best and then classified as preferred all of the other imagined headings for which the absolute angular error was within a certain range from that best heading. Because of the variability in overall performance and in the degrees to which the heading differences were pronounced for each participant, we did not specify the error range in absolute terms, but instead relative to the variability of each participant's performance on the different imagined headings. To avoid the problem that a participant with only one or two selected reference directions would, ceteris paribus, have a smaller standard deviation of the imagined heading means than would a participant who has selected four reference directions, we used the standard deviation of the four imagined headings with the worst performance for each participant. We then classified as preferred all of the imagined headings for which the participant's absolute angular error was within 1.5 standard deviations from the best heading, which resulted in an average of 3.8 reference directions per participant. The frequencies with which the eight headings were identified as preferred headings are reported in Table 1. As is evident in the table, the headings aligned with the geometry of the room were much more likely than other headings to be identified as reference directions. It is important to note that the data at the level of the individual participant are considerably more noisy than the combined data. Therefore, the estimated reference directions undoubtedly contain both false negatives and false positives, to a certain extent.

Table 1 Frequency with which each heading was identified as a reference direction in the participant-based analyses of Experiments 1 and 2

\begin{tabular}{lrrrrrrrrr}
\hline Experiment & $\mathrm{N}$ & $0^{\circ}$ & $45^{\circ}$ & $90^{\circ}$ & $135^{\circ}$ & $180^{\circ}$ & $225^{\circ}$ & $270^{\circ}$ & $315^{\circ}$ \\
\hline 1 & 32 & 25 & 8 & 25 & 8 & 23 & 4 & 21 & 8 \\
2 & 28 & 24 & 2 & 9 & 7 & 21 & 3 & 14 & 7 \\
\hline
\end{tabular}


Once we had estimated the reference directions for each participant, we categorized each trial for each participant into one of four categories: (1) both the imagined heading and the allocentric target direction corresponded to a preferred direction, (2) only the imagined heading corresponded to a preferred direction, (3) only the allocentric target direction corresponded to a preferred direction, and (4) neither of the two variables corresponded to a preferred direction. In the context of Fig. 5, with the assumption that all participants selected the aligned directions as reference directions, Category 1 corresponds conceptually to allocentric target directions of $0^{\circ}, 90^{\circ}, 180^{\circ}$, and $270^{\circ}$ for aligned headings (solid line); Category 2 corresponds conceptually to allocentric target directions of $45^{\circ}, 135^{\circ}, 225^{\circ}$, and $315^{\circ}$ for aligned headings (solid line); Category 3 corresponds conceptually to allocentric target directions of $0^{\circ}, 90^{\circ}, 180^{\circ}$, and $270^{\circ}$ for misaligned headings (dashed line); and Category 4 corresponds conceptually to allocentric target directions of $45^{\circ}$, $135^{\circ}, 225^{\circ}$, and $315^{\circ}$ for misaligned headings (dashed line). The resulting data were analyzed in a mixed-model ANOVA with terms for gender, study view $\left(0^{\circ}\right.$ or $\left.180^{\circ}\right)$, and headingtarget direction category (four levels), the latter of which was a within-participants variable. The mean angular errors for the four categories are presented in Table 2.

The main effect of heading-target direction category was significant, $F(3,84)=47.40, M S E=41.44, p<.001$ : Performance was better when both imagined heading and allocentric target direction corresponded to a preferred direction than when only imagined heading did, $F(1,84)=$ 13.69, MSE $=41.44, p<.001$, and performance was also better when only the imagined heading corresponded to a preferred direction than when only the allocentric target direction did, or when neither of the two did, $F \mathrm{~s}(1,84) \geq$ 35.12, $M S E=41.44, p \mathrm{~s}<.001$. Performance did not differ when only allocentric target direction corresponded to a preferred direction and when neither of the two did, $F(1$, $84)<1, M S E=41.44$. No other statistically reliable effects were obtained.

Table 2 Angular errors (in degrees) in judgments of relative direction for the four imagined heading-target direction categories in the participant-based analyses of Experiments 1 and 2

Correspondence to Reference Direction

\begin{tabular}{lllll}
\cline { 2 - 5 } Experiment & $\begin{array}{l}\text { Imagined } \\
\text { Heading } \\
\text { \& Target } \\
\text { Direction }\end{array}$ & $\begin{array}{l}\text { Imagined } \\
\text { Heading } \\
\text { Only }\end{array}$ & $\begin{array}{l}\text { Target } \\
\text { Direction } \\
\text { Only }\end{array}$ & $\begin{array}{l}\text { Neither } \\
\text { Imagined } \\
\text { Heading } \\
\text { nor Target } \\
\text { Direction }\end{array}$ \\
\hline 1 & 10.93 & 16.89 & 27.18 & 26.43 \\
2 & 11.75 & 18.94 & 29.87 & 33.05 \\
\hline
\end{tabular}

Reference directions were estimated for each participant individually from his or her pointing data

\section{Discussion}

Participants in both study view groups were able to recover the locations of objects more efficiently from the four imagined headings that were aligned with the dominant geometric structure of the environment (e.g., the walls of the room and the organization of the object layout) than from the misaligned imagined headings. This result suggests that the particular study conditions were conducive to participants in both groups predominantly representing the object layout using those four aligned directions as reference directions. This conclusion was supported by an analysis in which reference directions in memory were estimated for each participant individually.

The key finding of Experiment 1 was that those directions that were benefited when they were imagined headings (i.e., those directions that by conjecture were employed as reference directions) were also benefited as allocentric target directions: Participants were able to point to an object more accurately when the direction of the object was the same as one of the apparent reference directions, which lends support to the hypothesis that interobject spatial relations are preferentially represented when they coincide with a reference direction. Those allocentric target directions, however, were only benefited when participants imagined a heading that corresponded to a reference direction. For other imagined headings, no such effect of allocentric target direction was present. This suggests that determining the direction of a target object from such a heading is not independent of the inferential processes needed to recover the imagined heading.

It is important to note that the observed effect of allocentric target direction cannot be attributed to characteristics of the egocentric response method. When pointing to objects in aligned allocentric target directions from aligned imagined headings, the correct responses were front, back, left, and right. If the observed effect of allocentric target direction had been caused by participants finding it easier to indicate those directions with the joystick than diagonal ones, the same effect of allocentric target direction, only shifted in phase by $45^{\circ}$, should have been present for misaligned imagined headings, which was clearly not the case.

A potential limitation of Experiment 1 was that participants had predominantly represented the layout using the same four directions as reference directions and were therefore expected to perform well on those directions in allocentric target direction. An even stronger test of the hypothesis that interobject spatial relations are preferentially represented when they coincide with a reference direction would be possible if there were more variability in the number of reference directions that were selected by 
participants; it would then be possible to examine whether the benefited allocentric target directions varied as a function of the selected reference directions.

\section{Experiment 2}

Our aim in Experiment 2 was to attempt to create study conditions that would make it less likely that the majority of participants would represent the object layout relative to the four reference directions aligned with the dominant geometric structure of the environment. Participants in this experiment were also blindfolded, but were neither disoriented after entering the study room nor led on a convoluted path to their appropriate study position. We conjectured that because of the reduced need to reorient, participants might pay less attention to the geometry of the surrounding room, and therefore be less likely to select all four aligned directions as reference directions. The aim was to create more variability across participants in the number of reference directions selected.

\section{Method}

\section{Participants}

A group of 28 Vanderbilt University undergraduate and graduate students (14 female, 14 male) participated in this experiment in return for either credit toward a course requirement or $\$ 10-\$ 15$. One additional participant was excluded from the analyses because of excessive errors in pointing judgments.

\section{Materials and procedure}

The materials and the procedure of Experiment 2 were similar to those used in Experiment 1. The same object configuration was used, but the mat containing the objects was always placed at the lower end of the room (see Fig. 6). The mat location was varied for reasons unrelated to the present experiments, and the results of other experiments conducted in our laboratory indicated that it did not affect how participants represented the object layout. In contrast to Experiment 1, the participants were neither disoriented nor led on a convoluted path. Instead, they were led directly to the appropriate study position after being blindfolded. The trials presented were identical to those in Experiment 1.

\section{Results}

One participant exhibited an average absolute pointing error greater than $50^{\circ}$ and was excluded from the following analyses. Absolute pointing errors were analyzed in a mixed-model ANOVA with terms for gender, study view $\left(0^{\circ}\right.$ or $\left.180^{\circ}\right)$, imagined heading ( $\left(0^{\circ}\right.$ to $315^{\circ}$ in $45^{\circ}$ steps), and allocentric target direction $\left(0^{\circ}\right.$ to $315^{\circ}$ in $45^{\circ}$ steps). Imagined Heading and Allocentric Target Direction were within-participants factors. Because of the large number of effects, all tests were conducted at an alpha level of .01. Latencies showed the same general pattern as absolute pointing errors, indicating that there were no speed-accuracy trade-offs. In the interest of brevity, only the results for pointing errors are reported.

The main effect of imagined heading was significant, $F$ $(7,168)=15.47, M S E=621.28, p<.001$. The performance at various imagined headings was, however, not uniform across participants. Visual inspection of the data suggested that all participants performed well on the imagined headings of $0^{\circ}$ and $180^{\circ}$, but that participants differed in whether their performance was also improved on the $90^{\circ}$ and $270^{\circ}$ headings. For the following analyses, participants were divided into two groups using a median split based on the difference in their performance at the imagined headings of $0^{\circ}$ and $180^{\circ}$ versus $90^{\circ}$ and $270^{\circ}$. Because participants were grouped using data from these four imagined headings, we were primarily interested in effects that did not depend directly on relative levels of performance for those headings (e.g., effects of allocentric target direction and the relation between imagined heading and allocentric target direction for the two groups).

Figure 7A depicts absolute angular errors as a function of imagined heading and study view for those participants who were classified as having performed well only on the headings of $0^{\circ}$ and $180^{\circ}$. In both study view groups, these participants performed equally well on the imagined headings of $0^{\circ}$ and $180^{\circ}, F \mathrm{~s}(1,168)<1, M S E=621.28$, and their performance was better at $0^{\circ}$ and $180^{\circ}$ than at the other headings, $F \mathrm{~s}(1,168)$ $\geq 24.76, M S E=621.28, p \mathrm{~s}<.001$, including $90^{\circ}$ and $270^{\circ}, F \mathrm{~s}$ $(1,168) \geq 13.16, M S E=621.28, p \mathrm{~s}<.001$. Results from participants who were classified on the basis of the median split as having performed well on all four imagined headings that were aligned with the dominant geometric structure of the environment are plotted in Fig. 7B. In both study view groups, these participants performed significantly better at the aligned headings of $0^{\circ}, 90^{\circ}, 180^{\circ}$, and $270^{\circ}$ than at the misaligned headings of $45^{\circ}, 135^{\circ}, 225^{\circ}$, and $315^{\circ}, F \mathrm{~s}(1,168) \geq 17.44$, $M S E=621.28, p \mathrm{~s}<.001$, and for them, there was no advantage for $0^{\circ}$ and $180^{\circ}$ over $90^{\circ}$ and $270^{\circ}$ within the aligned headings, $F \mathrm{~s}(1,168)<1, M S E=621.28$.

The main effect of allocentric target direction was significant $F(7,168)=4.98, M S E=367.05, p<.001$, as was the interaction between allocentric target direction and imagined heading, $F(49,1176)=2.26, M S E=419.88, p<$ .001 . None of the effects involving study view group were significant, $p s \geq .060$; therefore, for the following analyses we collapsed across study views.

Figure 8A shows absolute pointing errors as a function of allocentric target direction and imagined heading for those 
Fig. 6 The two study view conditions used in Experiment

2. a $0^{\circ}$ study view; b $180^{\circ}$ study view. Participants were neither disoriented nor led on a convoluted path to their appropriate study position
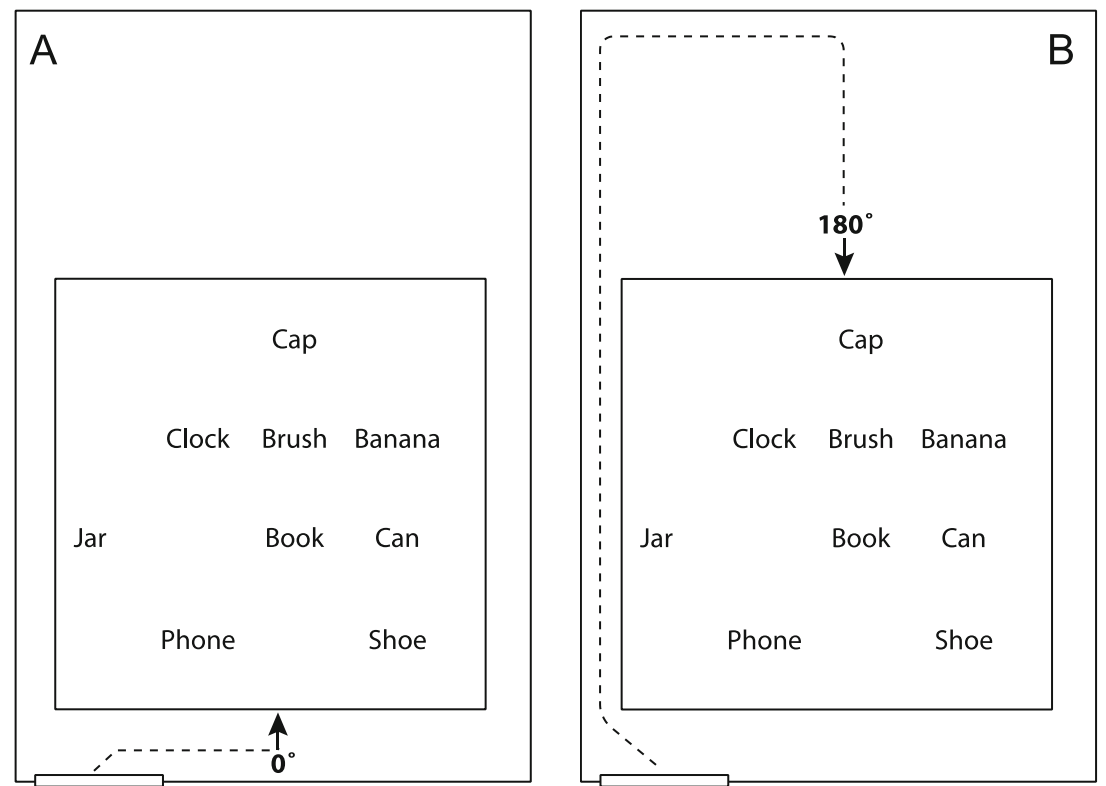

participants who, by hypothesis, selected only $0^{\circ}$ and $180^{\circ}$ as reference directions. These participants also showed a benefit for the allocentric target directions of $0^{\circ}$ and $180^{\circ}$, but only when imagining a heading of $0^{\circ}$ or $180^{\circ}, F(1,1344)=7.21$, $p<.01$ (lowest line in Fig. 8A). This effect of allocentric target direction was not present for the imagined headings of $90^{\circ}$ and $270^{\circ}, F(1,1344)=4.00, M S E=413.28, p=.046$, or for the misaligned imagined headings, $F(1,1344)=1.15, M S E=$ $413.28, p=.284$ (upper two lines in Fig. 8A). On average, even performance for the worst allocentric target directions at the imagined headings of $0^{\circ}$ and $180^{\circ}$ (i.e., allocentric target directions other than $0^{\circ}$ or $180^{\circ}$ ) was better than performance for all allocentric target directions at the remaining imagined headings, $F(1,1512)=63.09, M S E=436.39, p<.001$.

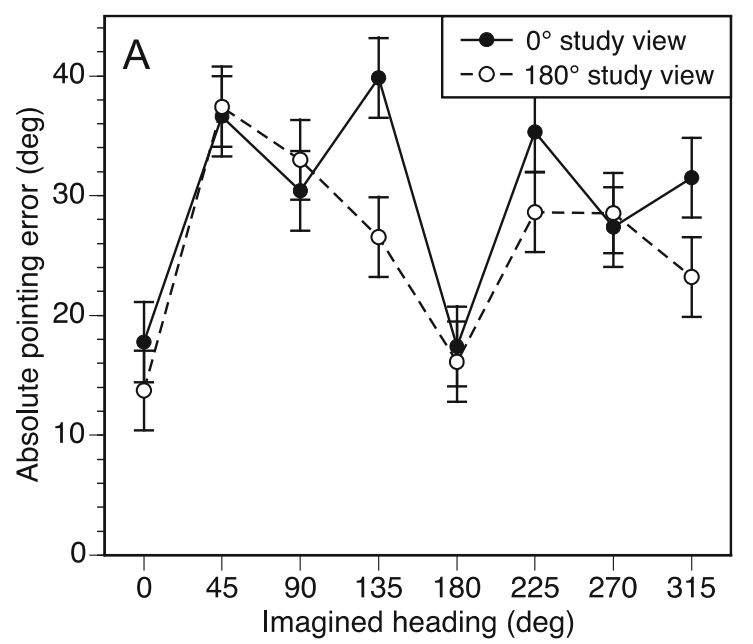

Fig. 7 Angular errors in judgments of relative direction as a function of imagined heading and study view in Experiment 2. Results are plotted separately for a participants who were classified as having only used $0^{\circ}$ and $180^{\circ}$ as reference directions and $\mathbf{b}$ participants who were
The results for allocentric target direction for those participants who had, by hypothesis, selected all four aligned directions as reference directions are plotted in Fig. 8B. These participants showed improved performance for the four aligned allocentric target directions, but only when they also imagined an aligned heading, $F(1,1344)=24.44, M S E=$ 413.28, $p<.001$. Within misaligned imagined headings, the difference between aligned and misaligned pointing directions was not significant, $F(1,1344)<1, M S E=413.28$. The performance for misaligned allocentric target directions within aligned headings was still better than performance for misaligned headings overall, $F(1,1512)=22.33, M S E=$ $436.39, p<.001$. No other statistically reliable effects were obtained.

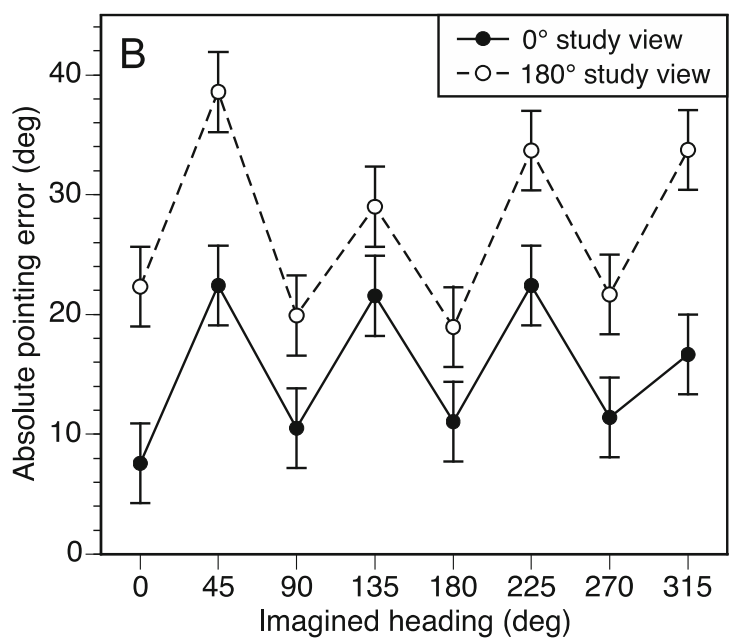

classified as having used $0^{\circ}, 90^{\circ}, 180^{\circ}$, and $270^{\circ}$ as reference directions. Error bars are confidence intervals corresponding to \pm 1 standard error of the mean, as estimated from the analysis of variance 


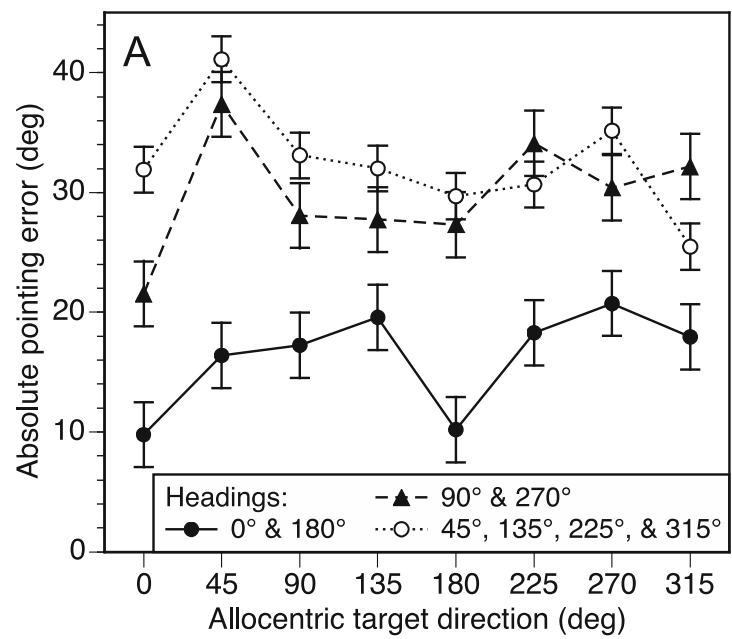

Fig. 8 Angular errors in judgments of relative direction as a function of allocentric target direction and imagined heading in Experiment 2. Results are plotted separately for a participants who were classified as having only used $0^{\circ}$ and $180^{\circ}$ as reference directions and $\mathbf{b}$ participants

The patterns of results for headings of $90^{\circ}$ and $270^{\circ}$ and for misaligned headings in Fig. 8A (upper two lines) and for misaligned headings in Fig. 8B (upper line) are quite similar: quadratic between $45^{\circ}$ and $270^{\circ}$, bracketed by somewhat better performance at $0^{\circ}$ and $315^{\circ}$. We cannot explain this effect, but suspect that it may be indicative of a real phenomenon, given that it appears in both subgroups of participants.

We conducted an additional analysis for Experiment 2, in which we estimated the selected reference directions at the level of individual participants, using the same methods as for Experiment 1. This analysis resulted in an average of 3.1 directions per participant that were classified as preferred. The frequencies with which the eight headings were identified as reference directions are reported in Table 1 . Note that $0^{\circ}$ and $180^{\circ}$ were identified as preferred headings by approximately the same proportions of participants in Experiments 1 and 2, whereas $90^{\circ}$ and $270^{\circ}$ were identified as preferred headings by greater proportions of participants in Experiment 1 than in Experiment 2. To analyze these effects statistically, we counted for each participant the numbers of preferred headings out of the two sets of interest $\left(0^{\circ}\right.$ and $180^{\circ}$ or $90^{\circ}$ and $270^{\circ}$ ), producing a variable that ranged from 0 to 2 for each participant, and then tested the mean difference on this variable between Experiments 1 and 2 for each set of headings. For $0^{\circ}$ and $180^{\circ}$, the average numbers of directions that were classified as preferred per participant did not differ between Experiment 1 and Experiment 2 (1.50 vs. 1.61, respectively), $t(58)<1, S E=0.147$; but for $90^{\circ}$ and $270^{\circ}$, the average numbers of directions that were classified as preferred per participant were higher in Experiment 1 than in Experiment $2(1.44$ vs. 0.82$), t(58)=3.11, S E=0.198, p<.01$. This pattern indicates that the study conditions of Experiment

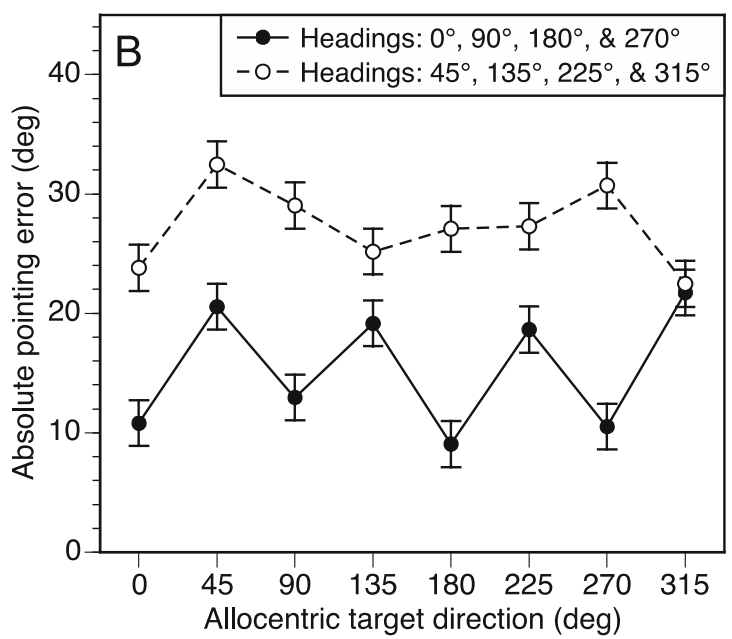

who were classified as having used $0^{\circ}, 90^{\circ}, 180^{\circ}$, and $270^{\circ}$ as reference directions. Error bars are confidence intervals corresponding to \pm 1 standard error of the mean, as estimated from the analysis of variance

2 successfully reduced participants' use of all four of the directions aligned with the geometry of the room as reference directions in memory.

Mean angular errors in pointing judgments are presented in Table 2. The data were analyzed in a mixed-model ANOVA with terms for gender, study view $\left(0^{\circ}\right.$ or $\left.180^{\circ}\right)$, and heading-target direction category (four levels), the latter of which was a within-participants variable. The main effect of heading-target direction category was significant, $F(3$, $72)=31.52, M S E=101.80, p<.001$ : Performance was better when both the imagined heading and allocentric target direction corresponded to a preferred direction than when only imagined heading did, $F(1,72)=7.10, M S E=101.80$, $p<.01$, and performance was better when only the imagined heading corresponded to a preferred direction than when only the allocentric target direction did or when neither of the two did, $F \mathrm{~s}(1,72) \geq 16.42, M S E=101.80, p \mathrm{~s}<.001$. Performance did not differ between when only allocentric target direction corresponded to a preferred direction and when neither of the two did, $F(1,72)=1.39, M S E=101.80$, $p=.242$. No other statistically reliable effects were obtained.

\section{Discussion}

The selection of reference directions in Experiment 2, as evidenced by performance at the various imagined headings, was not as uniform across participants as it was in Experiment 1 . Whereas some participants apparently represented the object layout relative to all four directions that were aligned with the dominant geometric organization of the environment, other participants represented the layout only relative to two of the aligned directions. The particular 
reference directions selected determined the nature of the effect of allocentric target direction. Participants who apparently selected four reference directions showed a benefit for those four directions in allocentric target direction, but participants who apparently only selected two reference directions showed a benefit in allocentric target direction for those two directions. In other words, the directions benefited in allocentric target direction varied systematically as a function of the reference directions selected, as hypothesized by the notion that interobject spatial relations are preferentially represented when they coincide with a reference direction. For both groups of participants, this effect of allocentric target direction, however, was only present when they imagined a heading that corresponded to a selected reference direction. No such effect was present for other imagined headings. An analysis in which reference directions in memory were identified for each participant individually supported these conclusions: Two of the directions aligned with the environmental geometry $\left(90^{\circ}\right.$ and $\left.270^{\circ}\right)$ were less likely to be identified as reference directions in Experiment 2 than in Experiment 1; the average number of preferred directions was lower in Experiment 2 (3.1) than in Experiment 1 (3.8) and corresponded to the average of the number of hypothesized reference directions in the group analyses (2 and 4); and pointing errors were lowest for those trials on which imagined heading and allocentric target direction both corresponded to a preferred direction.

\section{General discussion}

The goal of the experiments reported in this article was to test the hypothesis that interobject spatial relations are preferentially represented when they coincide with employed reference directions. The results of Experiment 1 supported this hypothesis by showing that participants were able to judge the relative direction of an object more effectively when the object was in one of the selected reference directions. A potential limitation of Experiment 1 was that there was little variability across participants in the selection of reference directions, making it difficult to determine whether differences in the number of reference directions selected would be manifested in allocentric target direction. Experiment 2 overcame this limitation by employing study conditions that were less conducive to representing the object layout with respect to the four directions aligned with the dominant geometric structure of the environment (e.g., the walls of the room and the organization of the object layout). This change appeared to have the effect of leading some participants to select four reference directions and other participants to select only two reference directions (corresponding to the study view and its opposite). The results showed that the particular reference directions selected determined which allocentric target directions were benefited, as predicted by the hypothesis that interobject spatial relations are preferentially represented when they coincide with employed reference directions. In both experiments, this effect of allocentric target direction, however, was only present for imagined headings that corresponded to one of the reference directions selected by a given participant. These findings were supported by additional analyses in which reference directions in memory were identified for each participant individually.

Our interpretation of these results is as follows. For imagined headings that correspond to a reference direction, pointing to objects that lie in a reference direction is comparatively easy, because the relative locations of those objects are represented with high probability or fidelity. Pointing from such headings to objects that do not lie in a reference direction is more difficult, because these relative object locations are represented only with lower fidelity or are not represented at all, and therefore must be inferred from the spatial relations that are explicitly represented. For imagined headings that do not correspond to a reference direction, interobject spatial relations generally need to be inferred. The mechanisms underlying these inferential processes remain unknown at this point. However, the nature of these processes is constrained to some extent by the finding that the effect of allocentric target direction was limited to imagined headings that corresponded to employed reference directions. This result indicates that the target direction and imagined heading are not retrieved or inferred independently. If these processes were independent, one would expect to see a benefit for allocentric target directions that coincided with a reference direction, even when the imagined headings did not (see also Roskos-Ewoldsen et al., 1998).

In both experiments, pointing judgments that were made from imagined headings that corresponded to a reference direction but for which the pointing direction did not coincide with a reference direction were better than pointing judgments that were made from imagined headings that did not correspond to a reference direction, regardless of the pointing direction. For example, in Fig. 5, the peaks of the function for aligned imagined headings are, with only one exception, below the function for misaligned imagined headings, and in Table 2, performance is better for trials on which only the imagined heading corresponded to a reference direction than for trials on which the imagined heading did not correspond to a reference direction. One possible explanation of this difference is that interobject spatial relations relative to a reference direction are represented, though with lower probability or fidelity, even if they do not coincide with a reference direction, whereas interobject spatial relations relative to nonreference directions are generally not represented (this notion is illustrated in Fig. 2). Another possibility is that when people imagine 
a heading that corresponds to a reference direction, they are able to use the represented spatial relations to readily infer required spatial relations that are not represented. For instance, in Fig. 2, the direction from 3 to 7 relative to $0^{\circ}\left(\overrightarrow{37}_{\alpha}\right)$ could be inferred from the represented spatial relations $\overrightarrow{34}_{\alpha}$ and $\overrightarrow{47}_{\alpha}$ (the latter is not depicted in the figure for legibility reasons), through a process analogous to vector addition. For imagined headings that do not correspond to a reference direction, this strategy would not work, because none of the represented spatial relations would be specified with respect to the imagined heading.

Our model in its existing form assumes that spatial relations are only represented with respect to selected reference directions, and that those spatial relations that are not represented need to be inferred, which results in observable costs. Another possibility is that spatial relations can be represented with respect to directions other than the dominant reference directions, although with lower probability. According to this perspective, impoverished performance on headings that do not correspond to reference directions would reflect a mixture of retrieval and costly inference. Alternatively (or perhaps in addition), spatial relations may also be represented with varying degrees of fidelity. In this case, impoverished performance would reflect the retrieval of low-fidelity spatial information. Determining the exact nature of the processes involved in making spatial judgments from nonpreferred headings will be an important goal of future research.

Another question that remains still unanswered concerns how exactly reference directions are selected. At this point, it is not entirely clear what factors determine, for instance, the number of reference directions selected. An important prerequisite for the selection of multiple reference directions seems to be that the selected directions be orthogonal to each other and that they correspond to salient directions in the environment (e.g., Mou et al., 2007). The saliency of a given direction in the environment, however, does not depend exclusively on properties of the environment, such as its geometrical structure and the organization of the to-berepresented objects, but also on how that direction is experienced. Egocentrically experiencing the direction or having the direction highlighted through instructions can make a direction more salient, and therefore more likely to be selected as a reference direction (e.g., Mou \& McNamara, 2002; Shelton \& McNamara, 1997). As the present experiments suggest, the need to completely reorient after disorientation may also increase the saliency of some directions, namely the ones that correspond to the boundaries of the enclosing space.

What the present experiments also demonstrate is that the selection of reference directions can vary considerably across participants. In Experiment 2, some participants performed equally well on the four imagined headings that were aligned with the dominant geometric structure of the environment, indicating that they had selected those four directions as reference directions, whereas other participants only performed well on the two imagined headings that were parallel with or opposite to their study view and did not show any benefit for the other two aligned headings. This finding illustrates the importance of examining performance not just at the level of collapsing across participants. Otherwise, performance patterns might be considered representative of all participants, when in fact they are only compounds of distinct performance patterns of subgroups of participants (e.g., Mou et al., 2007).

In summary, the present experiments provide support for the notion that interobject spatial relations are preferentially represented when they coincide with a utilized reference direction. A number of questions, however, remain unanswered. For instance, the nature of the effect of these preferences on the spatial representation is not known. Spatial relations that coincide with a utilized reference direction may be represented with higher probability, greater fidelity, or both. Other factors, in addition to coincidence with reference directions, may also affect the extent to which interobject spatial relations are represented. In an irregular layout or when a single reference direction is selected, not many spatial relations will coincide with a reference direction, and other factors will become influential. It is conceivable that proximity between the objects is one of these factors and that the spatial relation between two objects is represented with higher probability or fidelity when the two objects are closer together in space. In the present study, we did not vary interobject distance systematically. Another factor could be the salience of the reference object, so that the spatial relation between an object and a reference object is represented with higher probability or fidelity if the reference object is more salient (e.g., Chen \& McNamara, 2011). Shedding light on these questions will be an important goal of future research.

Author note Preparation of this article and the research reported in it were supported in part by National Institute of Mental Health Grant 2R01-MH57868 to T.P.M.

\section{References}

Burgess, N. (2008). Spatial cognition and the brain. Annals of the New York Academy of Sciences, 1124, 77-97.

Chen, X., \& McNamara, T. (2011). Object-centered reference systems and human spatial memory. Psychonomic Bulletin \& Review, 18, 985-991. doi:10.3758/s13423-011-0134-5

Cheng, K., \& Newcombe, N. S. (2005). Is there a geometric module for spatial orientation? Squaring theory and evidence. Psychonomic Bulletin \& Review, 12, 1-23. doi:10.3758/BF03196346 
Greenauer, N., \& Waller, D. (2008). Intrinsic array structure is neither necessary nor sufficient for nonegocentric coding of spatial layouts. Psychonomic Bulletin \& Review, 15, 1015-1021. doi:10.3758/PBR.15.5.1015

Holmes, M. C., \& Sholl, M. J. (2005). Allocentric coding of object-toobject relations in overlearned and novel environments. Journal of Experimental Psychology: Learning, Memory, and Cognition, 31, 1069-1087.

Kelly, J. W., McNamara, T. P., Bodenheimer, B., Carr, T. H., \& Rieser, J. J. (2008). The shape of human navigation: How environmental geometry is used in the maintenance of spatial orientation. Cognition, 109, 281-286.

Klatzky, R. L. (1998). Allocentric and egocentric spatial representations: Definitions, distinctions, and interconnections. In C. Freksa, C. Habel, \& K. F. Wender (Eds.), Spatial cognition: An interdisciplinary approach to representing spatial knowledge (pp. 1-17). Berlin: Springer.

Levinson, S. C. (1996). Frames of reference and Molyneux's question: Crosslinguistic evidence. In P. Bloom, M. A. Peterson, L. Nadel, \& M. F. Garrett (Eds.), Language and space (pp. 109-169). Cambridge: MIT Press.

McNamara, T. P. (2003). How are the locations of objects in the environment represented in memory? In C. Freksa, W. Brauer, C. Habel, \& K. F. Wender (Eds.), Spatial cognition III: Routes and navigation, human memory and learning, spatial representation and spatial reasoning, LNAI 2685 (pp. 174-191). Berlin: Springer.

McNamara, T. P., Rump, B., \& Werner, S. (2003). Egocentric and geocentric frames of reference in memory of large-scale space. Psychonomic Bulletin \& Review, 10, 589-595. doi:10.3758/BF03196519

Meilinger, T., \& Vosgerau, G. (2010). Putting egocentric and allocentric into perspective. In C. Hölscher, T. F. Shipley, M. O. Belardinelli, J. A. Bateman, \& N. S. Newcombe (Eds.), Spatial cognition VII (pp. 207-221). Berlin: Springer.

Mou, W., \& McNamara, T. P. (2002). Intrinsic frames of reference in spatial memory. Journal of Experimental Psychology: Learning, Memory, and Cognition, 28, 162-170.

Mou, W., McNamara, T. P., Rump, B., \& Xiao, C. (2006). Roles of egocentric and allocentric spatial representations in locomotion and reorientation. Journal of Experimental Psychology: Learning, Memory, and Cognition, 32, 1274-1290.

Mou, W., McNamara, T. P., Valiquette, C. M., \& Rump, B. (2004). Allocentric and egocentric updating of spatial memories. Journal of Experimental Psychology: Learning, Memory, and Cognition, 30, $142-157$.

Mou, W., Zhao, M., \& McNamara, T. P. (2007). Layout geometry in the selection of intrinsic frames of reference from multiple viewpoints. Journal of Experimental Psychology: Learning, Memory, and Cognition, 33, 145-154.

Pani, J. R., \& Dupree, D. (1994). Spatial reference systems in the comprehension of rotational motion. Perception, 23, 929-946.

Rock, I. (1973). Orientation and form. New York: Academic Press.

Roskos-Ewoldsen, B., McNamara, T. P., Shelton, A. L., \& Carr, W. (1998). Mental representations of large and small spatial layouts are orientation dependent. Journal of Experimental Psychology: Learning, Memory, and Cognition, 24, 215-226.

Sargent, J., Dopkins, S., Philbeck, J., \& Modarres, R. (2008). Spatial memory during progressive disorientation. Journal of Experimental Psychology: Learning, Memory, and Cognition, 34, 602-615.

Schmidt, T., \& Lee, E. Y. (2006). Spatial memory organized by environmental geometry. Spatial Cognition and Computation, 6 , 347-369.

Shelton, A. L., \& McNamara, T. P. (1997). Multiple views of spatial memory. Psychonomic Bulletin \& Review, 4, 102-106. doi:10.3758/BF03210780

Shelton, A. L., \& McNamara, T. P. (2001). Systems of spatial reference in human memory. Cognitive Psychology, 43, 274-310.

Steck, S. D., Mochnatzki, H. F., \& Mallot, H. A. (2003). The role of geographical slant in virtual environment navigation. In C. Freksa, W. Brauer, C. Habel, \& K. F. Wender (Eds.), Spatial cognition III: Routes and navigation, human memory and learning, spatial representation and spatial reasoning, LNAI 2685 (pp. 62-76). Berlin: Springer.

Tversky, B., Lee, P., \& Mainwaring, S. (1999). Why do speakers mix perspectives? Spatial Cognition and Computation, 1, 399-412.

Valiquette, C. M., McNamara, T. P., \& Smith, K. (2003). Locomotion, incidental learning, and the selection of spatial reference systems. Memory \& Cognition, 31, 479-489. doi:10.3758/BF03194405

Waller, D., \& Hodgson, E. (2006). Transient and enduring spatial representations under disorientation and self-rotation. Journal of Experimental Psychology: Learning, Memory, and Cognition, 32, $867-882$.

Wang, R. F., \& Spelke, E. S. (2000). Updating egocentric representations in human navigation. Cognition, 77, 215-250. 